\title{
NEARLY MODULAR ORTHOCOMPLEMENTED LATTICES
}

\author{
BY \\ M. DONALD MacLAREN
}

Introduction. Let $L$ be a complete, orthocomplemented lattice. We say that $L$ is a dimension lattice if $L$ is weakly modular and there is an equivalence relation on $L$ satisfying the axioms $\mathrm{A}, \mathrm{B}, \mathrm{C}$, and $\mathrm{D}^{\prime}$ of Loomis [5]. We say that $L$ is locally finite if every element of $L$ is the join of finite elements.

If $L$ is a dimension lattice in which every element is finite, then $L$ is modular. Conversely, Kaplansky [4] has shown that if $L$ is a complete orthocomplemented modular lattice, then $L$ is a continuous geometry. From this and the results of von Neumann [7] and Iwamura [3], it follows that $L$ is a dimension lattice in which every element is finite. Thus we conclude that $L$ is a finite dimension lattice if and only if $L$ is a complete orthocomplemented modular lattice. The main purpose of this paper is to obtain a similar characterization of locally finite dimension lattices.

To obtain such a characterization, we need to weaken the assumption that $L$ is modular. It is natural to try the assumption that $L$ is semimodular, but this is not enough. We need to know that in some sense enough modular pairs exist. For this reason, we define a modular element to be an element $a$ such that $[0, a]$ is a modular lattice and $(x, a)$ is a modular pair for all $x$ in $L\left(^{1}\right)$. An atom is always a modular element, and the finite elements in a dimension lattice are modular. We say that an orthocomplemented lattice $L$ is nearly modular, if $L$ is weakly modular and semi-modular and every element of $L$ is the join of modular elements. Our principal result is the following theorem $\left({ }^{2}\right)$.

THEOREM A. Let $L$ be a complete orthocomplemented lattice. Then $L$ is a locally finite dimension lattice if and only if $L$ is nearly modular.

In order to characterize the special class of Type I dimension lattices, we define an element $a$ to be minimal if for every $x \leqq a$ we can write $x=z a$ with $z$ in the center of $L$. Then we have

\footnotetext{
Received by the editors May 20, 1963.
}

( ${ }^{1}$ The author is indebted to G. W. Mackey for his suggesting the study of elements $a$ for which $(x, a)$ is always a modular pair.

( ${ }^{2}$ Ramsay [8] has proved independently a closely related theorem that $L$ is a locally finite dimension lattice if and only if $L$ contains a join-dense ideal which is itself a modular lattice. The connection between his work and ours will be discussed in $\$ 3$. 
THEOREM B. Let $L$ be a complete, weakly modular, orthocomplemented lattice. Then $L$ is a dimension lattice of Type $\mathrm{I}$ if and only if $L$ is semimodular, and there exists in $L$ a minimal element whose central cover is 1 .

The contents of the paper are as follows: In $\$ 1$ we summarize a few known definitions and results. In $\$ 2$ we develop the theory of modular elements. The key theorem is that under suitable conditions, if $a$ is modular and $b$ is perspective to $a$ then $b$ is modular. An alternate characterization of nearly modular lattices is given, and some continuity theorems involving modular elements are proved. Theorem $\mathrm{A}$ is proved in $\$ 3$ and Theorem $B$ in $\$ 4$. Finally, in $\$ 5$ we introduce a simple construction, the horizontal sum of two orthocomplemented lattices. This is used to give examples showing that neither of the conditions in the definition of nearly modular can be dropped.

1. Preliminaries about modular pairs. In this section, we give a few known definitions and results which will be used without explicit mention throughout this paper.

A pair of elements $(b, c)$ in a lattice $L$ is said to be a modular pair if for all $x \leqq c$ we have $(x \cup b) c=x \cup b c$. The pair $(a, b)$ is a $d$-modular pair if for all $x \geqq a$ we have $(a \cup b) x=a \cup b x$. $L$ is said to be semi-modular if the relation of being a modular pair is symmetric in $L$.

Lemma 1.1. Let $L$ be an orthocomplemented lattice. Then $(b, c)$ is a modular pair in $L$ if and only if $\left(c^{\prime}, b^{\prime}\right)$ is a d-modular pair.

COROLlary. $L$ is semi-modular if and only if the relation of being $a d$ modular pair is symmetric in $L$.

Following Loomis [5] we say that an orthocomplemented lattice is weakly modular if for $a \leqq b$ we have $b=a \cup a^{\prime} b$, that is, if $\left(a, a^{\prime}\right)$ is a $d$ modular pair for all $a$ in $L$. Some authors call a weakly-modular orthocomplemented lattice "orthomodular". From Lemma 3 of [5] we have the following useful result.

Lemma 1.2. Let $L$ be a weakly-modular orthocomplemented lattice. Then if $a \cup c \perp b$ we have $(a \cup b) c=a c$.

2. Modular elements. Let $a$ be an element in an orthocomplemented lattice $L$. We say that $a$ is modular if $[0, a]$ is a modular lattice and $(x, a)$ is a modular pair for all $x$ in $L$. We say that $a$ is $d$-modular if $[a, 1]$ is a modular lattice, and $(a, x)$ is a $d$-modular pair for all $x$ in $L$.

Lemma 2.1. Let $L$ be a semi-modular orthocomplemented lattice. The following statements about an element $a$ of $L$ are equivalent.

(1) $(x, a)$ is a modular pair for all $x$ in $L$.

(2) $\left(x, a^{\prime}\right)$ is a modular pair for all $x$ in $L$. 
(3) $(a, x)$ is a d-modular pair for all $x$ in $L$.

(4) $\left(a^{\prime}, x\right)$ is a d-modular pair for all $x$ in $L$.

Proof. From the fact that $L$ is orthocomplemented it follows immediately that (1) and (4) are equivalent and that (2) and (3) are equivalent. Since $L$ is semi-modular, (1) implies that $(a, x)$ is a modular pair for all $x$. But this means that $(x, a)$ is a $d$-modular pair for all $x$; and this is equivalent to (3), because $L$ is semi-modular. Thus (1) implies (3), and similarly (3) implies (1).

Lemma 2.2. The element $a$ is modular if and only if $a^{\prime}$ is d-modular.

Proof. The mapping $x \rightarrow x^{\prime}$ is an anti-isomorphism of $[0, a]$ onto $\left[a^{\prime}, 1\right]$. Thus $[0, a]$ is a modular lattice if and only if $\left[a^{\prime}, 1\right]$ is a modular lattice. By Lemma $2.1,(x, a)$ is a modular pair for all $x$ if and only if $\left(a^{\prime}, x\right)$ is a $d$-modular pair for all $x$.

Lemma 2.3. If $a$ is modular and $b \leqq a, b$ is modular. If $a$ is d-modular and $b \geqq a, b$ is $d$-modular.

Lemma 2.4. Suppose that $(a, x)$ is a d-modular pair for all $x$ in L. Then in the lattice [c,1], $(a \cup c, x)$ is a d-modular pair for all $x$.

Proof. Suppose that $c \leqq x$ and $a \cup c \leqq y$. Then $[(a \cup c) \cup x] y=(a \cup x) y$ $=a \cup x y=(a \cup c) \cup x y$.

Let $L$ be a complete orthocomplemented lattice, and let $a$ be an element such that $[0, a]$ is a modular lattice. We ask if $a$ is modular. In general the answer is no, even if $L$ is semi-modular. (See $\$ 5$ for an example.) The answer is, however, yes if there are enough modular elements in $L$. Because of this we introduce the following condition on an orthocomplemented lattice $L$.

$\left(^{*}\right)$ If $x \neq 0$ in $L$ then there exists $y \neq 0$ in $L$ such that $y \leqq x$ and $(z, y)$ is a modular pair for all $z$ in $L$.

Assuming this condition we can show that if $[0, a]$ is modular then $a$ is modular. The proof depends on Kaplansky's theorem [4] that a complete orthocomplemented modular lattice is a continuous geometry. We will use this frequently without explicit mention of it.

THEOREM 2.1. Let $L$ be a complete, weakly-modular, semi-modular orthocomplemented lattice for which condition $\left(^{*}\right)$ holds. Let a be an element of $L$. Then if $[a, 1]$ is a modular lattice, $a$ is d-modular. If $[0, a]$ is a modula: lattice, $a$ is modular.

Proof. It suffices to prove the first statement. Let $b \in L$. We will show that $(c, b)$ is a $d$-modular pair for all $c \geqq a$. Let $S$ be the set of $x \leqq b$ such that $(c, x)$ is a $d$-modular pair for all $c \geqq a$. Suppose that $\left\{x_{\alpha}\right\}$ is a chain in $S$. Let $y=\bigcup_{\alpha} x_{\alpha}$. Then for $a \leqq c \leqq d$, 


$$
(c \cup y) d=\left(c \cup \bigcup_{\alpha} x_{\alpha}\right) d=\left(\bigcup_{\alpha}\left(c \cup x_{\alpha}\right)\right) d \text {. }
$$

But $[a, 1]$ is a continuous geometry, so $\left[\bigcup_{\alpha}\left(c \cup x_{\alpha}\right)\right] d=\bigcup_{\alpha}\left[\left(c \cup x_{\alpha}\right) d\right]$ $=\bigcup_{\alpha}\left[c \cup x_{\alpha} d\right]=c \cup\left[\bigcup_{\alpha}\left(x_{\alpha} d\right)\right] \leqq c \cup y d$. Thus $(c, y)$ is a $d$-modular pair; $y$ is in $S$. By Zorn's lemma, $S$ contains a maximal element $x_{0}$. Let $y$ be an element such that $y \leqq x_{0}^{\prime} b$ and $(z, y)$ is a modular pair for all $z$ in $L$. We will show that $\left(c, x_{0} \cup y\right)$ is a $d$-modular pair for all $c \geqq a$. Since $x_{0}$ is maximal in $S$, this means that $y=0$. But it then follows from $\left(^{*}\right)$ that $x_{0}^{\prime} b=0$, i.e., that $b=x_{0}$; and this will prove the theorem. To show that $\left(c, x_{0} \cup y\right)$ is a $d$-modular pair for all $c \geqq a$, let $a \leqq c \leqq d$. Then

$$
\left(c \cup x_{0} \cup y\right) d \leqq\left[\left(c \cup x_{0}\right) \cup\left(y \cup x_{0}\right)\right]\left(x_{0} \cup d\right)=c \cup x_{0} \cup\left[\left(y \cup x_{0}\right)\left(x_{0} \cup d\right)\right]
$$

because, by Lemma 2.4, $\left(y \cup x_{0}, c \cup x_{0}\right)$ is a $d$-modular pair in $\left[x_{0}, 1\right]$, and $\left[x_{0}, 1\right]$ is semi-modular. Now $\left(y \cup x_{0}\right)\left(x_{0} \cup d\right)=x_{0} \cup d\left(y \cup x_{0}\right)$, because $\left(d, x_{0}\right)$ is a $d$-modular pair, and $L$ is semi-modular. Thus $\left(c \cup x_{0} \cup y\right) d$ $\leqq\left[c \cup x_{0} \cup d\left(y \cup x_{0}\right)\right] d=c \cup d\left(y \cup x_{0}\right) \cup x_{0} d$, because $\left(c \cup d\left(y \cup x_{0}\right), x_{0}\right)$ is a $d$-modular pair. Thus $\left(c \cup x_{0} \cup y\right) d \leqq c \cup d\left(y \cup x_{0}\right)$ for all $d \geqq c$, and this proves that $\left(c, x_{0} \cup y\right)$ is a $d$-modular pair for all $c \geqq a$.

LEMMA 2.5. Let $(c, d)$ be a pair of elements in an orthocomplemented lattice such that $(c, d)$ is both a modular pair and a $d$-modular pair and $c d=0$. Then the mapping $\theta: x \rightarrow x \cup c$ is an isomorphism of $[0, d]$ onto $[c, c \cup d]$.

Proof. If $\theta(x)=\theta(y)$, we have $x \cup c=y \cup c$. Therefore $(x \cup c) d=(y \cup c) d$. But $(x \cup c) d=x \cup c d=x$, and $(y \cup c) d=y$. Thus $x=y ; \theta$ is one-to-one. If $c \leqq y \leqq d \cup c, \theta(d y)=d y \cup c=(c \cup d) y=y$. Thus $\theta$ is onto. Clearly, $\theta(x) \leqq \theta(y)$ if and only if $x \leqq y$.

We will say that two elements $a$ and $b$ are perspective if there exists $c$ such that $a \cup c=b \cup c$ and $a c=b c=0$.

Theorem 2.2. Let L satisfy the hypotheses of Theorem 2.1. Let $c$ be a complement of $a$ in $L$. Then $a$ is modular if and only if $c$ is d-modular.

Proof. Suppose that $a$ is modular. Then $(a, c)$ is both a modular pair and a $d$-modular pair. By Lemma 2.5, $[0, a]$ and $[c, 1]$ are isomorphic. Thus $[c, 1]$ is a modular lattice. By Theorem $2.1, c$ is $d$-modular. A similar argument shows that if $c$ is $d$-modular, then $a$ is modular.

Corollary 1. If $a$ is modular, and $a \cup c=1$, then $c$ is $d$-modular. If $c$ is $d$-modular, and $a c=0$, then $a$ is modular.

Proof. Suppose that $a$ is modular and $a \cup c=1$. Let $d=(c a)^{\prime} c$. Then $d$ is a complement of $a$, so $d$ is $d$-modular. Since $d \leqq c, c$ is $d$-modular. The second part of the corollary is proved by a dual argument.

Corollary 2. If $a$ is modular, and $b$ is perspective to $a, b$ is modular. 
Proof. Since $a$ and $b$ are perspective, there exists $c$ such that $a \cup c=b \cup c$ and $a c=b c=0$. Let $d=c \cup(a \cup c)^{\prime}$. Then $d$ is a common complement of $a$ and $b$ [5, Proof of Lemma 38]. Since $a$ is modular, $d$ is $d$-modular, and therefore $b$ is modular.

We will denote the center of a lattice $L$ by $Z(L)$. The center of a complete, weakly-modular, orthocomplemented lattice $L$ is a complete Boolean sublattice of $L$ [ 1 , Corollaries to Theorems 6 and 7]. Therefore, for any $a$ in $L$, the intersection of all central elements $z \geqq a$ is itself a central element. It is called the central cover of $a$ and will be denoted by $e(a)$. The following characterization of central elements will be useful. The lemma is part of Theorem 3.2 of [6].

Lemma 2.6. Let a be an element in an orthocomplemented lattice $L$ such that $x=x a \cup x a^{\prime}$ for all $x$. Then $a \in Z(L)$.

CoROllary. Let a be an element in a weakly-modular orthocomplemented lattice $L$ such that $x a=0$ implies $x \perp a$. Then $a \in Z(L)$.

We will say that an orthocomplemented lattice $L$ is nearly modular if and only if $L$ is semi-modular and every element in $L$ is the join of modular elements. It is natural to ask, "Can this last condition be replaced by the condition that $L$ contains a modular element $a$ such that $e(a)=1$ ?" We suspect that the answer to this question is yes but have not been able to prove it. Statement (2) of the following theorem is the closest we have come to answering the question.

THEORM 2.3. Let $L$ be a complete, weakly-modular, orthocomplemented lattice. Then the following three statements about $L$ are equivalent.

(1) $L$ is nearly modular.

(2) $L$ is semi-modular, condition $\left(^{*}\right)$ holds for $L$, and there exists an element $c$ in $L$ such that $e(c)=1$ and $[0, c]$ is a modular lattice.

(3) The modular elements in L form a join-dense ideal.

Proof. Suppose that (1) holds. Let $\left\{a_{\alpha}\right\}$ be a maximal family of nonzero modular elements such that $e\left(a_{\alpha}\right) \perp e\left(a_{\beta}\right)$ for $\alpha \neq \beta$. Let $b=\bigcup_{\alpha} a_{\alpha}$. Then it is clear that $e(b)=1$, and $b$ is modular. Thus (2) holds. Now suppose that (2) holds, that $a$ and $b$ are modular, and that $x \leqq a \cup b$. Let $y=(x a)^{\prime} x$, $d=(b a)^{\prime} b$. Then $y a=d a=0$, and $y \cup a=(y \cup a)(a \cup d)=a \cup d(y \cup a)$. Thus $y$ and $d(y \cup a)$ are perspective. But $d \leqq b$, so $d(y \cup a)$ is modular. Thus $y$ is modular. Also $x a$ is modular. Now if $z \geqq x$ and $w$ is any element, we have $(x \cup w) z=(x a \cup y \cup w) z=x a \cup(y \cup w) z=x a \cup y \cup w z=x \cup w z$. Thus for every $x \leqq a \cup b$ and every $w,(x, w)$ is a $d$-modular pair. It follows that for every $x \leqq a \cup b, x$ is modular. Thus if (2) holds, the modular elements form an ideal. Let $a$ be the join of all modular elements in $L$. By Theorem 2.2 the element $c$, such that $[0, c]$ is a modular lattice and $e(c)=1$, is a modular element. Therefore $e(a)=1$. Suppose that $x$ is an 
element such that $x b=0$ for all modular elements $b$. Let $b$ be any modular element and let $d=b^{\prime}(x \cup b), x_{1}=(x d)^{\prime} x$. Let $y=\left(x_{1} \cup d\right) b$. Then $x_{1} d$ $=y d=0, \quad$ and $\quad y \cup d=\left(x_{1} \cup d\right) b \cup d=(d \cup b)\left(x_{1} \cup d\right)=\left(x_{1} \cup d\right)$. Thus $x_{1}$ and $y$ are perspective. But $y$ is modular, and $x_{1} \leqq x$. Hence $x_{1}=0$; $x=x d \leqq b^{\prime}$ for all modular $b$. This means that $x \leqq a^{\prime}$. It follows that for all $x$ in $L$, we have $x=x a \cup x a^{\prime}$. Therefore $a$ is in the center of $L$, and hence $a=1$. It now follows that if $x b=0$ for all modular $b$, then $x=0$. Thus every element $x$ is the join of modular elements. This completes the proof that (2) implies (3). Now Ramsay [8, Theorem 5.4] has shown that if a complete weakly-modular orthocomplemented lattice $L$ contains a join-dense ideal which is modular, then $L$ is semi-modular. It follows immediately that (3) implies (1).

We conclude this section with some continuity theorems involving modular elements. The first is a trivial consequence of Kaplansky's theorem. The other two depend on arguments using the property of being nearly modular.

THEOREM 2.4. Let $L$ be a complete, weakly-modular, orthocomplemented lattice, and let $c$ be any element of $L$. Then if $a$ is a modular element, and $\left\{b_{\alpha}\right\}$ is a chain such that $b_{\alpha} \leqq a$ for all $\alpha$, we have $c\left(\bigcup_{\alpha} b_{\alpha}\right)=\bigcup_{\alpha}\left(c b_{\alpha}\right)$. If $a$ is a d-modular element, and $\left\{b_{\alpha}\right\}$ is a chain such that $b_{\alpha} \geqq a$ for all $\alpha$, then $c \cup\left(\bigcap_{\alpha} b_{\alpha}\right)=\bigcap_{\alpha}\left(c \cup b_{\alpha}\right)$.

Proof. It suffices to prove the first statement. We have $c\left(\bigcup_{\alpha} b_{\alpha}\right)$ $=(c a)\left(\bigcup_{\alpha} b_{\alpha}\right)$. But $[0, a]$ is a continuous geometry, so $(c a)\left(\bigcup_{\alpha}^{\alpha} b_{\alpha}\right)$ $=\bigcup_{\alpha}\left(c a b_{\alpha}\right)=\bigcup_{\alpha}\left(c b_{\alpha}\right)$.

THEOREM 2.5. Let $L$ be a complete, nearly-modular, orthocomplemented lattice, and let $c$ be any element of $L$. Then if $a$ is modular, and $\left\{b_{\alpha}\right\}$ is a chain such that $b_{\alpha} \leqq a$ for all $\alpha$, we have $c \cup\left(\bigcap_{\alpha} b_{\alpha}\right)=\bigcap_{\alpha}\left(c \cup b_{\alpha}\right)$. If $a$ is $d$-modular, and $\left\{b_{\alpha}\right\}$ is a chain such that $b_{\alpha} \geqq a$ for all $\alpha$, we have $c\left(\bigcup_{\alpha} b_{\alpha}\right)=\bigcup_{\alpha}\left(c b_{\alpha}\right)$.

Proof. It suffices to prove the first statement. We need only show that $\bigcap_{\alpha}\left(b_{\alpha} \cup c\right) \leqq c \cup\left(\bigcap_{\alpha} b_{\alpha}\right)$. Suppose that $y$ is modular and $y \leqq \bigcap_{\alpha}\left(b_{\alpha} \cup c\right)$. Then for all $\alpha, y \leqq(y \cup a)\left(b_{\alpha} \cup c\right)=b_{\alpha} \cup c(y \cup a)$, because $b_{\alpha}$ is modular. Thus $y \leqq \bigcap_{\alpha}\left(b_{\alpha} \cup c(y \cup a)\right)$. But the lattice $[0, y \cup a]$ is a continuous geometry, so $\bigcap_{\alpha}\left(b_{\alpha} \cup c(y \cup a)\right)=c(y \cup a) \cup\left(\bigcap_{\alpha} b_{\alpha}\right) \leqq c \cup\left(\bigcap_{\alpha} b_{\alpha}\right)$. Thus for all modular $y \leqq \bigcap_{\alpha}\left(b_{\alpha} \cup c\right)$, we have $y \leqq c \cup\left(\bigcap_{\alpha} b_{\alpha}\right)$. Since the modular elements are join-dense in $L$, this proves that $\bigcap_{\alpha}\left(b_{\alpha} \cup c\right) \leqq c$ $\cup\left(\bigcap_{\alpha} b_{\alpha}\right)$.

TheOREM 2.6. Let $L$ be a complete, nearly-modular, orthocomplemented lattice, and let $\left\{b_{\alpha}\right\}$ be a chain of elements in $L$. Then if $a$ is modular, $a \cup\left(\bigcap_{\alpha} b_{\alpha}\right)=\bigcap_{\alpha}\left(a \cup b_{\alpha}\right)$. If $a$ is d-modular, $a\left(\bigcup_{\alpha} b_{\alpha}\right)=\bigcup_{\alpha}\left(a b_{\alpha}\right)$.

Proof. Again it suffices to prove the first statement, and we need only 
show that $\bigcap_{\alpha}\left(a \cup b_{\alpha}\right) \leqq a \cup\left(\bigcap_{\alpha} b_{\alpha}\right)$. Suppose that $x$ is a modular element, and that $x \leqq \bigcap_{\alpha}\left(a \cup b_{\alpha}\right)$. Then

$$
x \leqq \bigcap_{\alpha}\left[(a \cup x)\left(a \cup b_{\alpha}\right)\right]=\bigcap_{\alpha}\left[a \cup b_{\alpha}(a \cup x)\right]
$$

Now $\left\{b_{\alpha}(a \cup x)\right\}$ is a chain in the continuous geometry $[0, a \cup x]$, so $\bigcap_{\alpha}\left[a \cup b_{\alpha}(a \cup x)\right]=a \cup\left(\bigcap_{\alpha}\left[b_{\alpha}(a \cup x)\right]\right) \leqq a \cup\left(\bigcap_{\alpha} b_{\alpha}\right)$. Thus for all modular elements $x$ such that $x \leqq \bigcap_{\alpha}\left(a \cup b_{\alpha}\right)$, we have $x \leqq a \cup\left(\bigcap_{\alpha} b_{\alpha}\right)$. This proves that $\bigcap_{\alpha}\left(a \cup b_{\alpha}\right) \leqq a \cup\left(\bigcap_{\alpha} b_{\alpha}\right)$.

We might remark that the theorem "If a is modular, $a\left(\bigcup_{\alpha} b_{\alpha}\right)=\bigcup_{\alpha}\left(a b_{\alpha}\right)$," is not true. A counterexample is found by taking the element $a$ to be an atom in the lattice of closed subspaces of a Hilbert space.

3. A nearly-modular lattice is a locally finite dimension lattice. By a dimension lattice we will mean a complete, weakly-modular, orthocomplemented lattice on which there exists an equivalence relation satisfying the four axioms A, B, C, and $\mathrm{D}^{\prime}$ of Loomis [3]. These axioms are:

(A) If $a \sim 0$, then $a=0$,

(B) If $a_{1} \perp a_{2}$ and $b \sim a_{1} \cup a_{2}$, then there exists an orthogonal decomposition of $b, b=b_{1} \cup b_{2}$, such that $b_{1} \sim a_{1}$ and $b_{2} \sim a_{2}$,

(C) If $\left\{a_{\alpha}\right\}$ is a family of pairwise orthogonal elements, and if $\left\{b_{\alpha}\right\}$ is a second orthogonal family with the same indices such that $a_{\alpha} \sim b_{\alpha}$ for all $\alpha$, then $\bigcup_{\alpha} a_{\alpha} \sim \bigcup_{\alpha} b_{\alpha}$,

(D') If $a$ and $b$ have a common complement, then $a \sim b$.

An element $a$ in a dimension lattice is finite if $b \sim a$ and $b \leqq a$ implies $b=a$. A dimension lattice is locally finite if every element is the join of finite elements.

The purpose of this section is to prove Theorem A, i.e., to prove that a complete orthocomplemented lattice $L$ is a locally finite dimension lattice if and only if it is nearly modular. The "only if" part of the theorem is just a restatement of results of Ramsay [8, Theorem 5.4]. Before going on with the proof of the "if" part of the theorem, it seems appropriate to point out the relationship between our work and that of Ramsay [8]. Ramsay has proved that a complete, weakly-modular, orthocomplemented lattice $L$ is a locally finite dimension lattice if it contains a joindense modular ideal $I$. Thus we could combine his theorem with Theorem 2.3 to obtain our result. On the other hand he obtains as intermediate results the facts that the elements of $I$ are modular and that $L$ is semimodular. This shows that $L$ is nearly modular. The arguments of this section could, therefore, be applied to prove that $L$ is a locally finite dimension lattice. Ramsay's actual method of proof is different from ours. He works with a dimension function on $L$; we work directly with the equivalence relation. 
We now continue with the proof of Theorem A. Throughout the remainder of this section we will suppose that $L$ is a complete, nearlymodular, orthocomplemented lattice. We will write $a \approx b$ to mean that $a$ and $b$ are modular elements which are perspective. If $a$ is modular and $b \approx c \leqq a$, we will write $b \leqq a$. For two arbitrary elements $a$ and $b$ in $L$, we will write $a \sim b$ if and only if $e(a)=e(b)$ and for all $z$ in $Z(L)$ such that $z a$ or $z b$ is modular, we have $z a \approx z b$. If $a \sim b \leqq c$, we will write $a \prec c$. The remainder of this section will be devoted to proving that the relation $\sim$ is an equivalence relation and that it satisfies axioms $A, B, C$, and $D^{\prime}$. This will complete the proof of Theorem A. Note that axiom A follows immediately from the definition of $\sim$, for if $a \sim 0$, then $e(a)=0$, and hence $a=0$.

LEMmA 3.1. If $a$ and $b$ are perspective in an orthocomplemented lattice, then $e(a)=e(b)$.

Proof. This is Lemma 2.2 of [8].

Corollary 1. The relation $\sim$ satisfies axiom $\mathrm{D}^{\prime}$.

Proof. Suppose that $a$ and $b$ have a common complement $c$. By the lemma, $e(a)=e(b)$. Since central elements are distributive, we have for any $z \in Z(L)$ that $z c$ is an axis of perspectivity for $z a$ and $z b$.

Corollary 2. If $a \approx b$, then $a \sim b$.

Lemma 3.2. Let $a$ and $b$ be modular elements in $L$ such that $a \approx b$. Then there exists $d$ such that $a \cup b=a \cup d=b \cup d$ and $a d=b d=0$. Thus $a$ and $b$ are perspective in $a \cup b$.

Proof. Let $c$ be an axis of perspectivity for $a$ and $b$, and let $d=c(a \cup b)$. Then $(a \cup b)=(a \cup b)(a \cup c)=a \cup c(a \cup b)$, because $a$ is modular. Similarly, $a \cup b=b \cup d$. We have $a d=b d=0$, because $a c=b c=0$.

LEMмA 3.3. The relation $\approx$ is an equivalence relation on the set of modular elements in $L$. If $a$ is a modular element in $L$, then when it is restricted to the lattice $[0, a]$, the relation $\approx$ satisfies axioms $\mathrm{A}, \mathrm{B}, \mathrm{C}$, and $\mathrm{D}^{\prime}$.

Proof. The lemma is contained in Theorem 4.5 of [8]. We also note that it follows immediately from Kaplansky's theorem [4] and results of von Neumann [7] and Iwamura [3] on continuous geometries.

COROLlaRY. The relation $\sim$ is an equivalence relation on $L$.

Proof. Suppose that $a \sim b$ and $b \sim c$. We must show that $a \sim c$. Clearly $e(a)=e(c)$, and it suffices to prove that if $z \in Z(L)$ and $z a$ is modular, then $z a \approx z c$. Since $a \sim b, z b$ is modular and $z c \approx z b$. By the lemma, $\approx$ is transitive, so $z c \approx z a$.

Leмma 3.4. The relation $\sim$ is finitely additive. That is if $a_{1}, a_{2}, b_{1}, b_{2}$ 
are elements in $L$ such that $a_{1} \sim b_{1}, a_{2} \sim b_{2}, a_{1} \perp a_{2}$, and $b_{1} \perp b_{2}$, then $a_{1} \cup a_{2}$ $\sim b_{1} \cup b_{2}$.

Proof. Let $a=a_{1} \cup a_{2}$ and $b=b_{1} \cup b_{2}$. From elementary properties of central elements, it follows that $e(a)=e(b)$. Thus we need only show that if $z a$ or $z b$ is modular, then $z a \approx z b$; and we can assume that $z a$ is modular. Then $z a_{1}$ and $z a_{2}$ are both modular, and hence $z b_{1}$ and $z b_{2}$ are modular. Let $c=z a \cup z b=z a_{1} \cup z a_{2} \cup z b_{1} \cup z b_{2}$. Then $c$ is modular; and working in the dimension lattice $[0, c]$, we see that $z a \approx z b$.

In the next six lemmas we will prove that the relation $\sim$ is additive on chains, and axiom $\mathrm{C}$ will be obtained as a corollary. The essential step is the proof of Lemma 3.9 that if $a$ is modular and $\left\{b_{\alpha}\right\}$ is a chain such that $b_{\alpha} \lesssim a$ for all $\alpha$ then $\bigcup_{\alpha} b_{\alpha} \lesssim a$. This lemma will also be useful in the proof of axiom B. The key to proving Lemma 3.9 is to use the perspectivity theorem (2.2) and one of the continuity theorems (2.6) to reduce to the special case where $\bigcup_{\alpha} b_{\alpha} \perp a$. This special case is handled in Lemma 3.8. Lemmas 3.5 and 3.6 are simple statements about dimension lattices. The first follows from the additivity of the dimension function and the second from axiom $\mathrm{C}$ by a routine application of Zorn's lemma.

Lемма 3.5. Let $a$ and $b$ be finite elements in $a$ dimension lattice such that $a \leqq b$. Let $a_{1} \leqq a$ and $b_{1} \leqq b$ be such that $a_{1} \sim b_{1}$. Then $a_{1}^{\prime} a \leqq b_{1}^{\prime} b$. If $a \sim b$, then $a_{1}^{\prime} a \sim b_{1}^{\prime} b$.

Lemma 3.6. Let $\left\{a_{\alpha}\right\}$ and $\left\{b_{\alpha}\right\}$ be families in a dimension lattice, both having the same linearly ordered indexing set $I$, and such that $\alpha \leqq \beta$ implies $a_{\alpha} \leqq a_{\beta}$ and $b_{\alpha} \leqq b_{\beta}$. Assume further that $a_{\alpha} \sim b_{\alpha}$ for all $\alpha$ in I. Then $\bigcup_{\alpha} a_{\alpha}$ $\sim \bigcup_{\alpha} b_{\alpha}$.

LEMMA 3.7. Let $a$ be a modular element in $L$ and let $\left\{b_{\alpha}\right\}$ be a family of elements with linearly ordered indexing set I such that $\alpha \leqq \beta$ implies $b_{\alpha} \leqq b_{\beta}$ and such that $b_{\alpha} \lesssim a$ for all $\alpha$. Then there exists a family $\left\{a_{\alpha}\right\}$, also with indexing set $I$, such that $\alpha \leqq \beta$ implies $a_{\alpha} \leqq a_{\beta}, a_{\alpha} \approx b_{\alpha}$ for all $\alpha$, and $a_{\alpha} \leqq a$ for all $\alpha$.

Proof. Let $S$ consist of all pairs $\left\{J,\left\{x_{\alpha}\right\}\right\}$, where $J$ is a subset of $I$, and $\left\{x_{\alpha}\right\}$ is a family indexed by $J$ such that $\alpha \leqq \beta$ implies $x_{\alpha} \leqq x_{\beta}, x_{\alpha} \leqq a$ for all $\alpha$ in $J$, and $x_{\alpha} \sim b_{\alpha}$ for all $\alpha$ in $J$. Partially order $S$ by $\left\{J_{1},\left\{x_{\alpha}\right\}\right\}$ $\leqq\left\{J_{2},\left\{y_{\alpha}\right\}\right\}$ if and only if $J_{1} \subseteq J_{2}$ and $x_{\alpha}=y_{\alpha}$ for all $\alpha$ in $J_{1}$. Then clearly every chain in $S$ has an upper bound. Hence, by Zorn's lemma, $S$ contains a maximal element say $\left\{J,\left\{a_{\alpha}\right\}\right\}$. We need only prove that $J=I$. Suppose that this is not so. Then there exists $\beta$ in $I$ but not in $J$. Let

$$
x_{1}=\bigcup_{a_{\alpha}}(\alpha: \alpha<\beta, \alpha \in J), \quad y_{1}=\bigcup_{b_{\alpha}}(\alpha: \alpha<\beta, \alpha \in J) .
$$

Working in the dimension lattice $\left[0, a_{\beta} \cup b_{\beta}\right]$, we see that $x_{1} \approx y_{1} \leqq b_{\beta}$. If the set of $\alpha$ in $J$ such that $\alpha>\beta$ is nonvoid, let $x_{2}=\bigcap a_{\alpha}(\alpha: \alpha>\beta$ 
and $\alpha \in J$. Otherwise, let $x_{2}=a$. Since $\left[0, a \cup b_{\beta}\right]$ is a dimension lattice, it follows from Lemma 3.6 that $b_{\beta} \lesssim x_{2}$. Now from Lemma 3.5 we conclude that there exists $x_{3}$ such that $x_{1} \leqq x_{3} \leqq x_{2}$ and $b_{\beta} \approx x_{3}$. Therefore setting $a_{\beta}=x_{3}$ we can adjoin $\beta$ to $J$. This contradicts the maximality of $\left\{J,\left\{a_{\alpha}\right\}\right\}$ in $S$, thus proving that $J=I$.

LEMMA 3.8. Let a be a modular element in L. Let $\left\{b_{\alpha}\right\}$ be a family of elements in $L$ with the linearly ordered index set I such that $\alpha \leqq \beta$ implies $b_{\alpha}$ $\leqq b_{\beta}$, and such that $b_{\alpha} \leqq a$ and $b_{\alpha} \perp a$ for all $\alpha$. Then $b=\bigcup_{\alpha} b_{\alpha}$ is a modular element, and $b \leqq a$.

Proof. Let $\left\{a_{\alpha}\right\}, \alpha \in I$, be a family such that $\alpha \leqq \beta$ implies $a_{\alpha} \leqq a_{\beta}, a_{\alpha} \leqq a$ for all $\alpha$, and $a_{\alpha} \approx b_{\alpha}$ for all $\alpha$. We can assume that $a=\bigcup_{\alpha} a_{\alpha}$, and we then need only prove that $b$ is perspective to $a$. We can suppose that $I$ is infinite for otherwise the lemma is trivial. Let $c_{\alpha}=\left[\bigcup_{a_{\beta}}(\beta: \beta<\alpha)\right]^{\prime} a_{\alpha}$ and $d_{\alpha}=\left[\bigcup_{b_{\beta}}(\beta: \beta<\alpha)\right]^{\prime} b_{\alpha}$. Clearly, $a=\bigcup_{\alpha} c_{\alpha}$ and $b=\bigcup_{\alpha} d_{\alpha}$. Moreover, from the fact that for each $\alpha,\left[0, a_{\alpha} \cup b_{\alpha}\right]$ is a dimension lattice, we conclude that $c_{\alpha} \approx d_{\alpha}$ for all $\alpha$. Thus we have the following situation. There are two orthogonal families $\left\{c_{\alpha}\right\}$ and $\left\{d_{\alpha}\right\}$ such that $c_{\alpha} \approx d_{\alpha}$ for all $\alpha$. Moreover, $\bigcup_{\alpha} c_{\alpha} \perp \bigcup_{\alpha} d_{\alpha}$. We want to prove that $\bigcup_{\alpha} c_{\alpha}$ is perspective to $\bigcup_{\alpha} d_{\alpha}$. Since $\bigcup_{\alpha} c_{\alpha}$ and all the $d_{\alpha}$ are modular, the situation is very similar to that in the theory of continuous geometry where one wants to prove the corresponding theorem for independent sets of elements. We could obtain a straightforward proof along the lines of the proof of Theorem 3.6, part 1 of [7]. However, it happens that Holland [2] has proved a theorem which we can quote. He defines two elements $x$ and $y$ to be strongly perspective if there exists $z$ such that $x \cup y=x \cup z=y \cup z$ and $x z=y z=0$. His theorem [2, Theorem 1] states that if $L$ is a complete, weakly modular, orthocomplemented lattice and $\left\{x_{\alpha}\right\}$ and $\left\{y_{\alpha}\right\}$ are two families such that for $\alpha \neq \beta, x_{\alpha} \cup y_{\alpha} \perp x_{\beta} \cup y_{\beta}$ and such that for all $\alpha$, $x_{\alpha}$ and $y_{\alpha}$ are strongly perspective, then $\bigcup_{\alpha} x_{\alpha}$ and $\bigcup_{\alpha} y_{\alpha}$ are strongly perspective. In our case the families $\left\{c_{\alpha}\right\}$ and $\left\{d_{\alpha}\right\}$ clearly satisfy the orthogonality requirements of Holland's theorem, and, by Lemma 3.2, $c_{\alpha}$ is strongly perspective to $d_{\alpha}$ for each $\alpha$. Therefore $\bigcup_{\alpha} c_{\alpha}$ is strongly perspective to $\bigcup_{\alpha} d_{\alpha}$, i.e., $a$ and $b$ are strongly perspective.

LEMma 3.9. Let a be a modular element in L. Let $\left\{b_{\alpha}\right\}$ be a family with linearly ordered indexing set I such that $\alpha \leqq \beta$ implies $b_{\alpha} \leqq b_{\beta}$ and such that $b_{\alpha} \lesssim a$ for all $\alpha$. Then $b=\bigcup_{\alpha} b_{\alpha}$ is a modular element, and $b \leqq a$.

Proof. Let $c=a^{\prime} b$, and let $d=\left(a^{\prime} b\right)^{\prime} b$. Then $b=c \cup d$. We have $c$ $=a^{\prime}\left(\bigcup_{\alpha} b_{\alpha}\right)$. But $a^{\prime}$ is $d$-modular, and $\left\{b_{\alpha}\right\}$ is a chain, so, by Theorem 2.6, $c=\bigcup_{\alpha}\left(a^{\prime} b_{\alpha}\right)$. Clearly $a^{\prime} b_{\alpha} \lesssim a$ and $a^{\prime} b_{\alpha} \perp a$ for all $\alpha$. Therefore, by the preceding lemma, $c$ is a modular element. Now $a^{\prime}$ is $d$-modular and $d a^{\prime}$ $=0$. Therefore, $d$ is modular (Theorem 2.2). Thus $b$ is the join of two 
modular elements; $b$ is modular. Because $b$ is modular, $[0, a \cup b]$ is a dimension lattice, and we can apply Lemmas 3.7 and 3.6 to conclude that $b \lesssim a$.

LEMMA 3.10. Let $\left\{a_{\alpha}\right\}$ and $\left\{b_{\alpha}\right\}$ be families in $L$, both with the same linearly ordered indexing set $I$, such that $\alpha \leqq \beta$ implies $a_{\alpha} \leqq a_{\beta}$ and $b_{\alpha} \leqq b_{\beta}$. Assume further that $a_{\alpha} \sim b_{\alpha}$ for all $\alpha$. Then $\bigcup_{\alpha} a_{\alpha} \sim \bigcup_{\alpha} b_{\alpha}$.

Proof. Let $a=\bigcup_{\alpha} a_{\alpha}$ and $b=\bigcup_{\alpha} b_{\alpha}$. Clearly, $e(a)=e(b)$. Let $z$ be a central element such that $z a$ or $z b$ is modular. It is only necessary to prove that $z a \approx z b$, and we can assume that $z a$ is modular. Then $z a_{\alpha}$ is modular for all $\alpha$. Since $a_{\alpha} \sim b_{\alpha}$ for all $\alpha, z a_{\alpha} \approx z b_{\alpha}$ for all $\alpha$. Thus for all $\alpha, z b_{\alpha} \leqslant z a$. By the preceding lemma, $\bigcup_{\alpha}\left(z b_{\alpha}\right)$ is modular. Working in the dimension lattice $\left[0, z a \cup \bigcup_{\alpha}\left(z b_{\alpha}\right)\right]$ we conclude that $\bigcup_{\alpha}\left(z a_{\alpha}\right) \approx \bigcup_{\alpha}\left(z b_{\alpha}\right)$. But $z a$ $=\bigcup_{\alpha}\left(z a_{\alpha}\right)$ and $z b=\bigcup_{\alpha}\left(z b_{\alpha}\right)$, so the lemma is proved.

Corollary. The relation $\sim$ on $L$ satisfies axiom $\mathrm{C}$.

Proof. Let $\left\{c_{\alpha}\right\}$ and $\left\{d_{\alpha}\right\}$ be two orthogonal families with the same indexing set $I$ and such that $c_{\alpha} \sim d_{\alpha}$ for all $\alpha$. We must prove that $\bigcup_{\alpha} c_{\alpha} \sim \bigcup_{\alpha} d_{\alpha}$. Well order the set $I$, and let $a_{\alpha}=\bigcup_{\beta \leqq \alpha} c_{\beta}$ and $b_{\alpha}=\bigcup_{\beta \leqq \alpha} d_{\beta}$. We claim that $a_{\alpha} \sim b_{\alpha}$ for all $\alpha$. Suppose that this is not so. Then there exists a smallest $\beta$ such that " $a_{\beta} \sim b_{\beta}$ " does not hold. Let $x=\bigcup_{a_{\alpha}}(\alpha: \alpha<\beta)$ and $y=\bigcup b_{\alpha}(\alpha: \alpha<\beta)$. By the lemma, $x \sim y$. Moreover, $a_{\beta}=c_{\beta} \bigcup x$, $b_{\beta}=d_{\beta} \cup y, c_{\beta} \perp x, d_{\beta} \perp y$, and $c_{\beta} \sim d_{\beta}$. Therefore by Lemma 3.4, $a_{\beta} \sim b_{\beta}$, which is a contradiction. Now clearly $\bigcup_{\alpha} c_{\alpha}=\bigcup_{\alpha} a_{\alpha}$ and $\bigcup_{\alpha} d_{\alpha}=\bigcup_{\alpha} b_{\alpha}$. By the lemma, $\bigcup_{\alpha} a_{\alpha} \sim \bigcup_{\alpha} b_{\alpha}$, so the corollary is proved.

To complete the proof of Theorem A, we must show that axiom B holds. Suppose for the moment that $Z(L)$ is trivial. Then any two nonmodular elements are equivalent. Let $a \sim b$, and $a=a_{1} \cup a_{2}$, where $a_{1} \perp a_{2}$. We seek an orthogonal decomposition $b=b_{1} \cup b_{2}$ such that $b_{1} \sim a_{1}$ and $b_{2} \sim a_{2}$. If $a$ is modular, such a decomposition obviously exists. If $a_{1}$ is modular but $a_{2}$ is not, we find $b_{1} \leqq b$ such that $b_{1} \sim a_{1}$. Then $b_{1}^{\prime} b$ is not modular, and so $b_{1}^{\prime} b \sim a_{2}$. If neither $a_{1}$ nor $a_{2}$ is modular, we need only split $b$ into two nonmodular elements.

The proof in the general case, where $Z(L)$ is nontrivial, proceeds along the same lines. However, to take care of nonmodular elements which are not equivalent, the notion of a purely infinite element is introduced. We will say that the element $a \in L$ is purely infinite if and only if whenever $z \in Z(L)$ and $z a$ is modular then $z a=0$. From the definition of a purely infinite element and the equivalence relation we get immediately the following lemma.

LEMMA 3.11. If $a$ and $b$ are both purely infinite and $e(a)=e(b)$, then $a \sim b$. If $a$ is purely infinite and $b \sim a$, then $b$ is purely infinite. 
We will say that two elements $a$ and $b$ of $L$ are related if there exist modular elements $a_{1} \leqq a$ and $b_{1} \leqq b$ such that $a_{1} \sim b_{1} \neq 0$. It follows from the definition of the relation $\sim$ that if $e(a) \perp e(b)$ then $a$ and $b$ are not related. We need the converse of this, and it comes as a corollary to the following theorem, which is of some interest in itself.

THEOREM 3.1. Let $a$ be an element in the complete, nearly-modular, orthocomplemented lattice $L$. Then the center of $[0, a]$ is just the set $\{z a: z \in Z(L)\}$.

Proof. Since the modular elements of $L$ form an ideal, this theorem is contained in Theorem 4.3 of [8]. The author independently obtained a proof using results of von Neumann [7].

COROLlary. If $a$ and $b$ are not related, $e(a) \perp e(b)$.

Lemмa 3.12. Let $a \neq 0$ be purely infinite. Then $a$ has an orthogonal decomposition $a=a_{1} \cup a_{2}$ such that $e\left(a_{1}\right)=e\left(a_{2}\right)$ and both $a_{1}$ and $a_{2}$ are purely infinite.

Proof. Let $w$ be a modular element such that $w \leqq a$. We will show that there exists a modular element $x \leqq w^{\prime} a$ such that $x \sim w$. Let $S$ be the set of all modular elements $y \leqq w^{\prime} a$ such that $y \lesssim w$. It follows from Lemma 3.9 that every chain in $S$ has an upper bound. Hence $S$ has a maximal element $x_{0}$, and for some $x_{1} \leqq w, x_{0} \sim x_{1}$. Suppose that $x_{1}<w$. Then $\left(x_{0} \cup w\right)^{\prime} a$ is not related to $x_{1}^{\prime} w$, for otherwise $x_{0}$ would not be maximal in $S$. It follows that $\left.e\left(x_{1}^{\prime} w\right)\left[x_{0} \cup w\right)^{\prime} a\right]=0$, so we have

$$
e\left(x_{1}^{\prime} w\right) a=e\left(x_{1}^{\prime} w\right)\left(x_{0} \cup w\right) .
$$

But $e\left(x_{1}^{\prime} w\right)\left(x_{0} \cup w\right)$ is modular and $a$ is purely infinite. Therefore $e\left(x_{1}^{\prime} w\right) a$ $=0$. This contradicts the assumption that $x_{1}<w$. Thus $x_{1}=w ; x_{0} \sim w$. Now we can construct the orthogonal decomposition of $a$. Let $c_{1} \leqq a$ be a modular element, such that $e\left(c_{1}\right)=e(a)$. Let $d_{1}<c_{1}^{\prime} a$ be a modular element such that $d_{1} \sim c_{1}$. Let $b_{1}=0$. Construct by induction sequences of elements $\left\{c_{n}\right\},\left\{d_{n}\right\},\left\{b_{n}\right\}$ such that $b_{n+1}=c_{1} \cup d_{1} \cup \cdots \cup c_{n} \cup d_{n}, c_{n} \leqq b_{n}^{\prime} a$, $c_{n} \sim b_{n}, d_{n} \leqq\left(c_{n} \cup b_{n}\right)^{\prime} a, d_{n} \sim c_{n} \cup b_{n}$. Let $a_{1}=\bigcup_{n} c_{n}, a_{2}=\bigcup_{n} d_{n}$. If $z \in Z(L)$ and $z a_{1} \neq 0$, we have $z c_{1} \neq 0$, because $e\left(c_{1}\right)=e\left(a_{1}\right)$. Then for all $n, z c_{n}$ $>z c_{1} \neq 0$. It follows that $z a_{1}$ is not modular. Thus $a_{1}$ is purely infinite. Similarly, $a_{2}$ is purely infinite. By their construction, $a_{1} \perp a_{2}$, and $e\left(a_{1}\right)$ $=e\left(a_{2}\right)=e(a)$.

LEMMA 3.13. Let $a=a_{1} \cup a_{2}$, where $a_{1} \perp a_{2}, a_{1}$ is modular, and $a_{2}$ is purely infinite. Let $b$ be an element such that $b \sim a$. Then there exists an orthogonal decomposition of $b, b=b_{1} \cup b_{2}$, such that $b_{1} \sim a_{1}$, and $b_{2} \sim a_{2}$.

Proof. Let $S$ consist of all elements $x \leqq b$ such that $x \leqq a_{1}$. Then if $\left\{x_{\alpha}\right\}$ is a chain in $S, \bigcup_{\alpha} x_{\alpha}$ is in $S$ by Lemma 3.9. Thus $S$ contains a maximal element $b_{1}$. Let $b_{2}=b_{1}^{\prime} b$. We will first show that $z a_{2}=0$ if and only if 
$z b_{2}=0$. Suppose that $z a_{2}=0$. Then $z a=z a_{1}$, so $z a_{1} \sim z b_{1} \cup z b_{2}$. Let $b_{3}$ $=b_{1} \cup z b_{2}$. Then $z b_{3}=z b_{1} \cup z b_{2} \sim z a_{1}$ and $z^{\prime} b_{3}=z^{\prime} b_{1} \prec z^{\prime} a_{1}$. Thus $b_{3} \prec a_{1}$. Since $b_{1}$ was maximal in $S$ this means $b_{3}=b_{1}$, i.e., $z b_{2}=0$. Suppose on the other hand that $z b_{2}=0$. Then $z b=z b_{1}$ and $z b \approx z a_{1} \cup z a_{2}$. Thus $z a_{2}$ is modular; $z a_{2}=0$ because $a_{2}$ is purely infinite. Now it follows immediately that $e\left(a_{2}\right)=e\left(b_{2}\right)$. Further, if $z a_{2}$ is modular, $z a_{2}=0$, so $z b_{2}=0 ; z a_{2} \approx z b_{2}$. If $z b_{2}$ is modular, $z b$ is modular, which means $z a_{2}=0$. This gives $z b_{2}=0$, so again $z a_{2} \approx z b_{2}$. Thus $a_{2} \sim b_{2}$. Now suppose that $b_{1} \sim c_{1}<a_{1}$. Then $c_{1}^{\prime} a_{1}$ is not zero. But $c_{1}^{\prime} a_{1}$ is unrelated to $b_{2}$, for otherwise $b_{1}$ would not be maximal in $S$. Taking $z=e\left(c_{1}^{\prime} a\right)$, this gives $z \perp b_{2}$ and $z \perp a_{2}$. Hence $z a=z a_{1}, \quad z b=z b_{1}$, and $z a \sim z b$. This gives $z b_{1} \sim z a_{1}=z c_{1} \cup z c_{1}^{\prime} a_{1}=z c_{1}$ $\cup c_{1}^{\prime} a_{1}$. But $z c_{1} \sim z b_{1}$, and $a_{1} \cup b_{1}$ is modular, so we must have $c_{1}^{\prime} a_{1}=0$. This contradiction proves that $a_{1} \sim b_{1}$.

LEMma 3.14. The relation $\sim$ on $L$ satisfies axiom $B$.

Proof. Let $a=a_{1} \cup a_{2}$ and let $b \sim a$. We must show that there exists an orthogonal decomposition of $b, b=b_{1} \cup b_{2}$, such that $b_{1} \sim a_{1}$ and $b_{2} \sim a_{2}$. Let $e_{1}$ be the join of all central elements $z \leqq e(a)$ such that $z a$ is modular. Note that $e_{1} a$ is modular. Let $e_{2}$ be the join of all central elements $z \leqq e_{1}^{\prime} e(a)$ and such that $z a_{1}$ is modular. Then $e_{2} a_{1}$ is modular and $e_{2} a_{2}$ is purely infinite. Let $e_{3}$ be the join of all central elements $z \leqq\left(e_{1} \cup e_{2}\right)^{\prime} e(a)$ such that $z a_{2}$ is modular. Then $e_{3} a_{2}$ is modular and $e_{3} a_{1}$ is purely infinite. Finally, let $e_{4}=\left(e_{1} \cup e_{2} \cup e_{3}\right)^{\prime} e(a)$. Then $e_{4} a_{1}$ and $e_{4} a_{2}$ are both purely infinite. It is now clear from the preceding results that for $i=1,2,3,4$ the element $e_{i} b$ has an orthogonal decomposition $e_{i} b=x_{i} \cup y_{i}$ such that $x_{i} \sim e_{i} a_{1}$ and $y_{i} \sim e_{i} a_{2}$. Taking $b_{1}=\bigcup_{i} x_{i}$ and $b_{2}=\bigcup_{i} y_{i}$, we get the desired decomposition of $b$.

This completes the proof of Theorem A.

4. Minimal elements and Type I dimension lattices. Let $L$ be an orthocomplemented lattice. We will say that an element $a \in L$ is minimal if for every $x \leqq a$ there exists $z \in Z(L)$ such that $x=z a$. If $L$ is modular this definition is equivalent to von Neumann's definition of minimal elements in a continuous geometry. (See [7, p. 277, especially Lemma 3.2].) Clearly, if $a$ is minimal, $[0, a]$ is a Boolean algebra. We remark that if $L$ is complete and nearly modular, and $[0, a]$ is a Boolean algebra, then $a$ is minimal. This follows from Theorem 3.1 .

Leмma 4.1. Let a be a minimal element in a complete, weakly-modular, orthocomplemented lattice $L$. Then $a$ is modular.

Proof. Let $x \leqq a$, and let $y$ be any element of $L$. There exists $z \in Z(L)$ such that $z a=x$. We have then $(x \cup y) a=\left(z a \cup z y \cup z^{\prime} y\right)\left(z a \cup z^{\prime} a\right)$ $=z(a \cup y) a \cup\left(z^{\prime}(a y)\right)=z a \cup z a y \cup z^{\prime} a y=x \cup y a$. Thus $(y, a)$ is a mod- 
ular pair for all $y$. We know that $[0, a]$ is a Boolean algebra. Therefore $a$ is modular.

THeOREM 4.1. Let $L$ be a complete, semi-modular, weakly-modular, orthocomplemented lattice. Let a be a minimal element of $L$. Then if $b$ is perspective to $a, b$ is minimal.

Proof. Let $c$ be an axis of perspectivity for $a$ and $b$. Let $d=c^{\prime}(a \cup c)$. Then $d$ and $a$ are perspective with axis $c$. Since $a$ is modular, $(c, a)$ is both a modular pair and a $d$-modular pair. Further, $d$ is the orthocomplement of $c$ in the weakly-modular orthocomplemented lattice $[0, a \cup c]$. Thus $(c, d)$ is both a modular pair and a $d$-modular pair in $[0, a \cup c]$. Let $\theta$ be the map from $[0, a]$ to $[0, d]: \theta(x)=(x \cup c) d$. By Lemma $2.5, \theta$ is onto. Let $\phi$ be the map of $Z(L)$ into $[0, d]: \phi(z)=\theta(z a)$. Since $a$ is minimal, the $\operatorname{map} z \rightarrow z a$ is onto. Therefore $\phi$ is onto. Now

$$
\phi(z)=(z a \cup c) d=\left(z a \cup z c \cup z^{\prime} c\right)\left(z d \cup z^{\prime} d\right)=z(a \cup c) d \cup z^{\prime} c d=z d .
$$

Thus the map $z \rightarrow z d$ from $Z(L)$ to $[0, d]$ is onto; $d$ is minimal. To prove that $b$ is minimal we carry out a similar argument using the fact that $c$ is an axis of perspectivity for $b$ and $d$. The only point to note is that $c$ is the orthocomplement in $[0, a \cup c]$ of the modular element $d$. Thus in $[0, a \cup c]$, $(c, b)$ is both a modular pair and a $d$-modular pair.

THEOREM 4.2. Let $L$ be a complete, semi-modular, weakly-modular, orthocomplemented lattice. Suppose further that $L$ contains a minimal element a such that $e(a)=1$. Then every element is the join of minimal elements, and hence $L$ is nearly modular.

Proof. Let $d$ be the join of all minimal elements, and suppose that $c$ is an element such that $c x=0$ for all minimal elements $x$. For $x$ minimal let $y=x^{\prime}(x \cup c)$ and $c_{1}=(c y)^{\prime} c$. Then $c_{1} \cup y=\left(c_{1} \cup y\right)(x \cup y)=y \cup x\left(c_{1} \cup y\right)$. Clearly, $c_{1} y=\left[x\left(c_{1} \cup y\right)\right] y=0$. Thus $c_{1}$ and $x\left(c_{1} \cup y\right)$ are perspective. Therefore $c_{1}$ is minimal, and this means $c_{1}=0$. This proves that if $c x=0$ for all minimal $x, c \perp x$. Thus if $c d=0, c \perp d$. Therefore, $d \in Z(L)$, and every element $y \leqq d$ is the join of minimal elements. But by hypothesis there exists a minimal element $a$ such that $e(a)=1$. Therefore $d=1$, and hence every element is the join of minimal elements.

Let us recall a few definitions from [5]. Two elements $a$ and $b$ in a dimension lattice $L$ are said to be related if there exist $a_{1} \leqq a$ and $b_{1} \leqq b$ such that $a_{1} \sim b_{1} \neq 0$. An element $a$ is simple if $x \cup y \leqq a$ and $x \perp y$ imply that $x$ is not related to $y$. The dimension lattice $L$ is of Type $I$ if 1 is the join of simple elements. Theorem $B$, which we restate below for the reader's convenience, gives a natural characterization of Type I dimension lattices. It is somewhat more pleasing than the theorem for arbitrary locally finite dimension lattices, because we need only assume that one 
minimal element with central cover 1 exists, rather than requiring that every element be the join of minimal elements.

THEOREM B. Let $L$ be a complete weakly-modular orthocomplemented lattice. Then $L$ is a dimension lattice of Type I if and only if $L$ is semimodular and there exists in $L$ a minimal element whose central cover is 1 .

Proof. Suppose that $L$ is semi-modular and contains a minimal element $a$ such that $e(a)=1$. Then by the preceding theorem, $L$ is nearly modular and hence is a locally finite dimension lattice. Moreover, every element in $L$ is the join of minimal elements. But if $b$ is a minimal element, $[0, b]$ is a Boolean algebra. Therefore if $x \cup y \leqq b$ and $x y=0$, then $x$ and $y$ are not related by the perspectivity relation. Thus every minimal element is simple, and $L$ is of Type I. Suppose now that $L$ is a Type I dimension lattice. Then $L$ is locally finite and hence is semi-modular. In $L$ the element 1 is the join of simple elements. Hence there exists a simple element $a$ such that $e(a)=1$. We need only show that $a$ is minimal. By Theorem 3 of [5] there exists for any $x \leqq a$ an invariant element $z$ such that $z a=x$. (The element $z$ is invariant if $z$ and $z^{\prime}$ are unrelated.) By Theorem 2 of [5], $z$ invariant implies $y=y z \cup y z^{\prime}$ for all $z$. Therefore if $z$ is invariant, $z \in Z(L)$. Thus for any $x \leqq a$, there exists $z \in Z(L)$ such that $z a=x$. That is, $a$ is minimal.

5. Horizontal sums and examples. Let $L_{1}$ and $L_{2}$ be two orthocomplemented lattices which have no elements in common, except perhaps 0 and 1. Let $L$ be the set union of $L_{1}$ and $L_{2}$ with the two zero elements identified and with the two unit elements identified. We can introudce a partial ordering in $L$ by $x \leqq y$ if and only if for some $i$ both $x$ and $y$ are in $L_{i}$ and $x \leqq y$ in $L_{i}$. Then $L$ is an orthocomplemented lattice. If $x \in L_{i}$, the orthocomplement of $x$ in $L$ is just the orthocomplement of $x$ in $L_{i}$. We call $L$ the horizontal sum of $L_{1}$ and $L_{2}$ and write $L=L_{1} \circ L_{2}$. If $x$ and $y$ are different from 0 and $1, x \in L_{1}$, and $y \in L_{2}$, then in $L_{1} \circ L_{2}$ the elements $x$ and $y$ are not related by the partial ordering. The next theorem follows trivially from this fact.

THEOREM 5.1. Let $L=L_{1} \circ L_{2}$, where $L_{1}$ and $L_{2}$ are orthocomplemented lattices. Then $L$ is complete if and only if $L_{1}$ and $L_{2}$ are both complete. $L$ is weakly modular if and only if $L_{1}$ and $L_{2}$ are both weakly modular.

Now let $L_{1}$ and $L_{2}$ be complete, modular, orthocomplemented lattices which contain no atoms. For example, let $L_{1}$ be the lattice of projections in a factor of Type $\mathrm{II}_{1}$. Then $L_{1} \circ L_{2}$ is semi-modular, but $L_{1} \circ L_{2}$ is not nearly modular. If $a$ is different from 0 and 1 in $L_{1} \circ L_{2}$, then $[0, a]$ is a modular lattice, but $a$ is not modular.

Let $L_{1}$ and $L_{2}$ be complete, weakly-modular, atomic, orthocomplemented 
lattices, and suppose that $L_{1}$ contains at least three orthogonal atoms. Then every element in $L_{1} \circ L_{2}$ is the join of atoms and hence of modular elements, but $L_{1} \circ L_{2}$ is not semi-modular. Also it is easily verified that $L_{1} \circ L_{2}$ is irreducible. It follows that for any atom $a$ in $L_{1} \circ L_{2}, e(a)=1$. Thus $L_{1} \circ L_{2}$ contains a minimal element whose central cover is 1 .

\section{REFERENCES}

1. S. S. Holland, Jr., A Radon-Nikodym theorem in dimension lattices, Trans. Amer. Math. Soc. 108 (1963), 66-87.

2. _ Analysis of weakly modular lattices, Notices Amer. Math. Soc. 8 (1961), 432.

3. Tsurane Iwamura, On continuous geometry. I, Japan J. Math. 19 (1944), 57-71.

4. Irving Kaplansky, Any orthocomplemented complete modular lattice is a continuous geometry, Ann. of Math. (2) 61 (1955), 524-541.

5. L. H. Loomis, The lattice theoretic background of the dimension theory of operator algebras, Mem. Amer. Math. Soc. No. 18 (1955).

6. M. D. MacLaren, Atomic orthocomplemented lattices, Pacific J. Math. i4 (1964), 597-612.

7. J. von Neumann, Continuous geometry, Princeton Mathematical Series, No. 25, Princeton Univ. Press, Princeton, N. J., 1960.

8. Arlan Ramsay, Dimension theory in an arbitrary complete orthomodular lattice, Trans. Amer. Math Soc. (to appear).

\section{Boeing Company,}

Seattle, Washington 